\title{
Prevalence and outcomes of incidental imaging findings: umbrella review
}

\author{
Jack W O'Sullivan, ${ }^{1}$ Tim Muntinga, ${ }^{1}$ Sam Grigg, ${ }^{2}$ John P A loannidis $3,4,5,6,7$
}

${ }^{1}$ Centre for Evidence-Based Medicine, Nuffield Department of Primary Care Health Sciences, University of Oxford, Oxford

OX2 6GG, UK

${ }^{2}$ University of Melbourne, Victoria, Australia

${ }^{3}$ Stanford Prevention Research Center, Stanford University School of Medicine, Stanford, CA, USA

${ }^{4}$ Department of Health Research and Policy, Stanford University School of Medicine, Stanford,

CA, USA

${ }^{5}$ Department of Biomedical Data Science, Stanford University School of Medicine, Stanford, CA, USA

${ }^{6}$ Department of Statistics, Stanford University School of Humanities and Sciences, Stanford, CA, USA

${ }^{7}$ Meta-Research Innovation Center at Stanford (METRICS), Stanford University, Stanford, CA, USA

Correspondence to:

J W O'Sullivan

jack.osullivan@phc.ox.ac.uk (or@DrjackOSullivan on Twitter; ORCID 0000-0003-3629-2546)

Additional material is published online only. To view please visit the journal online.

Cite this as: BMJ 2018;361:k2387 http://dx.doi.org/10.1136/bmj.k2387

Accepted: 29 April 2018

\section{ABSTRACT}

OBJECTIVE

To provide an overview of the evidence on prevalence and outcomes of incidental imaging findings.

DESIGN

Umbrella review of systematic reviews.

\section{DATA SOURCES}

Searches of MEDLINE, EMBASE up to August 2017; screening of references in included papers.

\section{ELIGIBILITY CRITERIA}

Criteria included systematic reviews and meta-analyses of observational studies that gave a prevalence of incidental abnormalities ("incidentalomas"). An incidental imaging finding was defined as an imaging abnormality in a healthy, asymptomatic patient or an imaging abnormality in a symptomatic patient, where the abnormality was not apparently related to the patient's symptoms. Primary studies that measured the prevalence of incidentalomas in patients with a history of malignancy were also considered in sensitivity analyses.

\section{RESULTS}

20 systematic reviews ( 240 primary studies) were identified from 7098 references from the database search. Fifteen systematic reviews provided data to quantify the prevalence of incidentalomas, whereas 18 provided data to quantify the outcomes of incidentalomas (13 provided both). The prevalence of incidentalomas varied substantially between imaging tests; it was less than $5 \%$ for chest computed tomography for incidental pulmonary embolism in patients with and without cancer and whole body positron emission tomography (PET) or PET/computed tomography (for patients with and without cancer). Conversely, incidentalomas occurred in more than a third of images in cardiac magnetic resonance

\section{WHAT IS ALREADY KNOWN ON THIS TOPIC}

Imaging scans are increasingly performed, and imaging resolution continues to improve

Thus, "incidentalomas" (that is, incidental abnormalities) are increasingly common

Estimates concerning the prevalence and outcomes of incidentalomas vary

\section{WHAT THIS STUDY ADDS}

The prevalence as well as outcomes of incidentalomas (including the metaanalysed estimates of malignancy rate) can now be quantified, stratified by imaging scan and organ

Data will aid clinicians and patients weigh up the pros and cons of requesting imaging scans and assist with management decisions after an incidentaloma diagnosis

These results can underpin the creation of guidelines to assist these decisions imaging (MRI), chest computed tomography (for incidentalomas of thorax, abdomen, spine, or heart), and computed tomography colonoscopy (for extracolonic incidentalomas). Intermediate rates occurred with MRI of the spine (22\%) and brain (22\%). The rate of malignancy in incidentalomas varied substantially between organs; the prevalence of malignancy was less than $5 \%$ in incidentalomas of the brain, parotid, and adrenal gland. Extra-colonic, prostatic, and colonic incidentalomas were malignant between $10 \%$ and $20 \%$ of the time, whereas renal, thyroid, and ovarian incidentalomas were malignant around a quarter of the time. Breast incidentalomas had the highest percentage of malignancy $(42 \%, 95 \%$ confidence interval $31 \%$ to $54 \%$ ). Many assessments had high between-study heterogeneity (15 of 20 metaanalyses with $\mathrm{I}^{2}>50 \%$ ).

\section{CONCLUSIONS}

There is large variability across different imaging techniques both in the prevalence of incidentalomas and in the prevalence of malignancy for specific organs. This umbrella review will aid clinicians and patients weigh up the pros and cons of requesting imaging scans and will help with management decisions after an incidentaloma diagnosis. Our results can underpin the creation of guidelines to assist these decisions.

\section{SYSTEMATIC REVIEW REGISTRATION}

PROSPERO: CRD42017075679.

\section{Introduction}

Incidentalomas, incidental imaging findings serendipitously diagnosed in an asymptomatic patient or symptomatic patient undergoing imaging for an unrelated reason, ${ }^{1-3}$ are fast becoming a modern medical crisis. ${ }^{4}$ The rapid rise in demand for imaging, ${ }^{56}$ coupled with rapidly advancing image resolution is driving a surge in incidentalomas. ${ }^{7-9}$

Incidentalomas are problematic because they can lead to patient anxiety. ${ }^{10}$ Given the clinical uncertainty surrounding their ideal management, they can encourage further investigations and potentially overtreatment. In response to the risks of incidentalomas, a US Presidential Commission encouraged clinicians to discuss with patients incidentalomas as a risk of imaging before they order a scan. ${ }^{4}$ Despite this, informed consent before imaging remains poor. ${ }^{11}$ It is difficult for clinicians to appropriately inform patients of the chance of incidental findings because the data quantifying prevalence is inconsistent and unclear.

Clinicians have also expressed uncertainty about the ideal management of incidentalomas, particularly primary care physicians. ${ }^{312}$ Although some guidelines 
exist, ${ }^{13-17}$ they are often directed at radiologists ${ }^{15-17}$ and for many organs (eg, colon, spine, breast, brain, prostate, and parotid) there are no guidelines to address the management of incidentalomas.

Several systematic reviews have been published exploring the prevalence and outcomes of incidentalomas. These studies use inconsistent and often inappropriate synthesis methods, and commonly only focus on one imaging scan or organ. We set out to conduct an umbrella review of all existing systematic reviews, meta-analysing data with appropriate methods and including data for all imaging scans and organs. We aimed to quantify the prevalence with which incidentalomas emerge on any type of imaging test and quantify the outcomes of incidentalomas stratified by organ. We thus hoped to provide both clinicians and policy makers with robust data in an easy-to-access and inclusive format to inform clinical practice and guidelines. The availability of data from diverse imaging modalities and diverse imaging areas and organs in an umbrella review should provide a more comprehensive overview of the available evidence.

\section{Methods \\ Protocol, registration, and study design \\ The protocol for this umbrella review was developed and registered a priori (PROSPERO: CRD42017075679). We conducted an umbrella review of systematic reviews and meta-analyses of observational studies describing the prevalence and outcomes of incidental imaging findings (incidentalomas).}

\section{Search strategy and eligibility criteria}

Using a structured search strategy (appendix 1), we searched MEDLINE and EMBASE databases up to August 2017. Two authors (TM, SG) independently undertook a three-step parallel review of title, abstract, and full text screens based on a predefined

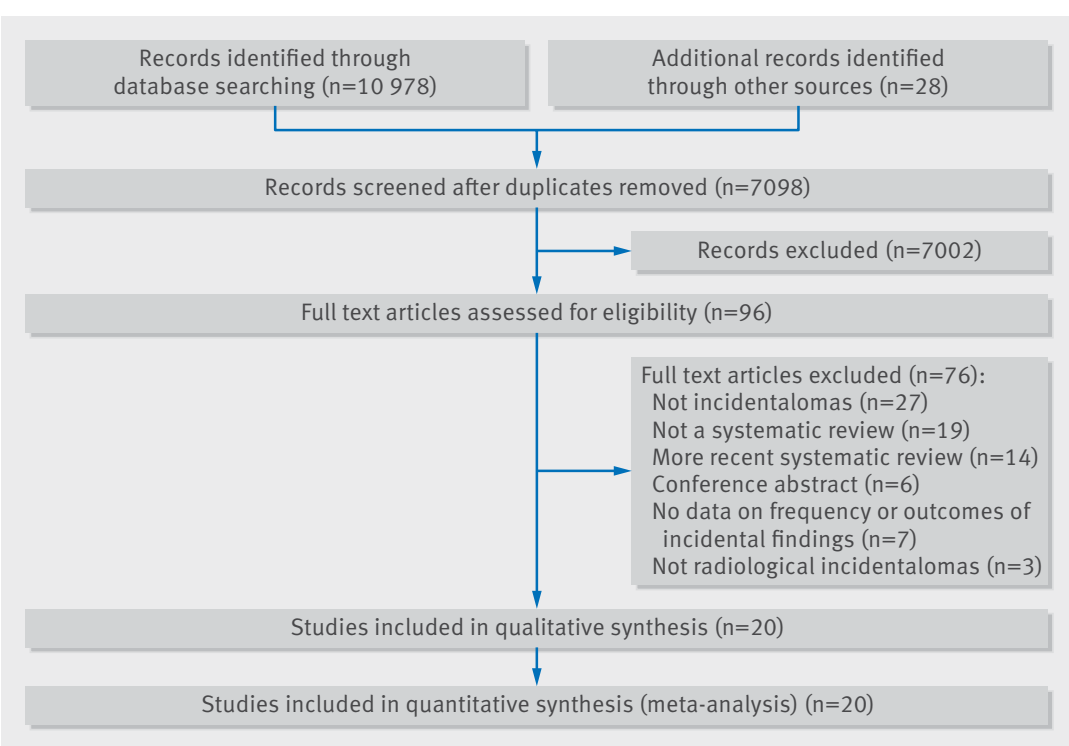

Fig 1 | PRISMA flow diagram inclusion and exclusion criteria (fig 1). Disagreements were resolved by a third author (JWOS). We included systematic reviews and meta-analyses of observational studies that gave a prevalence of incidental abnormalities (incidentalomas). An incidental imaging finding was defined as an imaging abnormality in a healthy, asymptomatic patient or in a symptomatic patient, where the abnormality was not apparently related to the patient's symptoms. ${ }^{1-3}$ For instance, an adrenal lesion noted in a patient who is undergoing computed tomography of the abdomen after a traumatic accident. Primary studies that measure the detection of incidentalomas in patients with a history of malignancy were included, but only within further sensitivity analyses, because incidentalomas in these patients could often represent metastases (see Sensitivity analyses section below). Study designs beyond systematic reviews and meta-analyses were excluded. Further, systematic reviews and metaanalyses without explicit, systematic literature searches and those that did not inform the prevalence or outcomes of incidentalomas were excluded (as consistent with previous umbrella reviews ${ }^{18}{ }^{19}$ ). We also excluded studies that quantified non-imaging incidentalomas (eg, surgical incidentalomas) and conference abstracts (we were unable to complete second level data extraction on conference abstracts).

\section{Data extraction}

We undertook data extraction on two levels. The first level concerned extraction from included systematic reviews and meta-analyses. The second level included the extraction of data from the primary studies within the included systematic reviews and meta-analyses.

For first level extraction, we extracted PubMed ID, lead author's name, publication year, study population, average age of population, number of studies included, organ of incidental findings, imaging modality, number of imaging studies, number of incidental findings, number of incidental findings with definitive diagnosis, eg, proportion of incidental findings that were ultimately diagnosed as a malignancy (separately for each type of definitive diagnosis). When we found more than one systematic review or meta-analysis quantifying incidentalomas for the same imaging modality and organ, we included the largest and most recent meta-analysis, consistent with previous umbrella reviews. ${ }^{18} 19$ If the most recent meta-analysis was not the largest, we explored this discrepancy and compared the included primary studies of both reviews, identifying the primary studies missing from the most recent systematic review. We then extracted and included the data from these primary studies missing from the most recent systematic review, to be inclusive.

For the second level extraction, we extracted from the primary studies within included systematic reviews: lead author's name, study design, organ of incidentaloma, imaging modality, number of patients undergoing imaging studies, number of patients with identified incidentalomas, number of incidentalomas 
with definitive diagnosis (separately for each type of definitive diagnosis). One author (JWOS) extracted data, which was checked by a second author (SG).

\section{Statistical analysis \\ Data analysis}

We conducted separate meta-analyses for the percentage of patients undergoing imaging with at least one incidentaloma (prevalence), and the percentage of incidentalomas that were malignant (outcome). We grouped and meta-analysed data by imaging modality and body organ for prevalence of incidentalomas (eg, prevalence of incidentalomas from magnetic resonance imaging (MRI) of the spine). For these analyses, the numerator was the number of patients who had at least one incidentaloma and the denominator was the total number of patients who underwent imaging studies. Thus, we only counted one incidentaloma per patient, which was consistent with most of the primary research. For meta-analyses concerning the rate of malignancy from incidentalomas, we metaanalysed data for each organ and thus the numerator was the number of incidentalomas with a diagnosis of malignancy and the denominator was the total number of incidentalomas. Studies of patients with a history of malignancy were not included in the primary analyses, as incidentalomas in these patients could often represent metastasis, but these studies were included in the sensitivity analysis (see Sensitivity analyses below).

Given the substantial variation in methods used by the included systematic reviews to summarise results, we conducted our own meta-analyses of incidentaloma prevalence and malignancy rate. All meta-analyses used a random effects model ${ }^{20}$ with corresponding $95 \%$ confidence intervals. Given that our included data are percentages, we performed double arcsine transformation to stabilise the variance, ${ }^{21}$ using the inverse variance method. ${ }^{22}$ For each meta-analysis, along with summary estimates, we estimated several metrics including: heterogeneity with the $\mathrm{I}^{2}$ metric (considered large for values $>50 \%$ and very large for values of $>75 \%)^{23}$ and predictive intervals (to predict the range of prevalence that would be expected in a new study/new population). ${ }^{2425}$ We also estimated the between-study variance $\left(\tau^{2}\right)$.

\section{Sensitivity analyses}

We conducted sensitivity analyses including primary studies that measured the prevalence and outcomes of incidental findings in patients with a history of malignancy. Some literature extends its definition of incidentalomas to include patients with a history of malignancy who are asymptomatic or do not have a history of disease in the organ of interest. For example, a thyroid lesion noted incidentally in a patient with a history of colorectal cancer and without symptoms or a history of thyroid disease.

Further, in meta-analyses with $\mathrm{I}^{2}$ values greater than $50 \%$ not explained by meta-regression (see Quality assessment and meta-regression section below) we conducted post hoc sensitivity analyses excluding up to one outlying study (study furthest from the summary estimate) and then reassessed the heterogeneity $\left(\mathrm{I}^{2}\right){ }^{26}$

\section{Quality assessment, meta-regression, and overall} assessment of the evidence

Two authors (JWOS, SG) independently assessed each included systematic review and meta-analysis using the Assessing the Methodological Quality of Systematic Reviews (AMSTAR) tool. ${ }^{27}$ Previous literature has shown that lower sample sizes are associated with a higher prevalence of incidentaloma malignancies. ${ }^{28}$ To explore this across the included meta-analyses, we used random effects meta-regression to explore whether the estimate of the prevalence was associated with the size of the study (the denominator in the proportion). The meta-regressions were initially weighted by each study's variance and then adjusted by the random-effects variance in the final model.

The amount and consistency of the evidence was judged based on whether the total evidence for each analysis included at least 1000 incidentalomas and whether the heterogeneity $\mathrm{I}^{2}$ was less than $50 \%$. Other criteria used for assessment of credibility in umbrella reviews $^{181929}$ are not relevant here, given that our assessed data refers to prevalence rather than associations.

\section{Patient involvement}

No patients were involved in the study design or conduct, nor the interpretation of results. We plan on disseminating our results through the patient networks within the University of Oxford and also, funding allowing, gather the thoughts of how our results can help patients make better shared decision regarding imaging and incidental findings.

\section{Results}

Study selection and characteristics

We included 20 systematic reviews from 7098 references identified from our database search (fig 1). ${ }^{7-9} 28$ 30-45 These 20 systematic reviews included 240 primary studies and 627073 patients. Fifteen systematic reviews provided data to quantify the prevalence of detecting incidentalomas ${ }^{7-9} 28$ 30-39 45 (table 1), whereas 18 provided data to quantify the

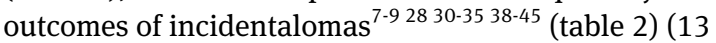
provided both).

\section{Prevalence of incidentalomas}

We conducted nine meta-analyses quantifying the prevalence of incidentalomas across each individual imaging test (appendix 2). The median number of studies included in meta-analyses was 11 (range 3-93), the median number of participants was 14409 (557$197386)$, and the median number of incidentalomas was 998 (24-4922). Three of our meta-analyses addressed incidentalomas in MRI scans (spine, cardiac, and brain), four for computed tomography (three for chest and one for computed tomography colonoscopy), and two for positron emission tomography (PET) or 


\begin{tabular}{|c|c|c|c|c|c|c|}
\hline $\begin{array}{l}\text { Systematic review } \\
\text { (lead author, year) }\end{array}$ & $\begin{array}{l}\text { No of included } \\
\text { studies }\end{array}$ & Population & Organ of incidentaloma & Imaging modality & $\begin{array}{l}\text { No of } \\
\text { patients }\end{array}$ & $\begin{array}{l}\text { No of patients with } \\
\text { incidental findings }\end{array}$ \\
\hline Nayan 2014 & 30 & $\begin{array}{l}\text { Oncology patients ( } n=17) \text {, asymptomatic } \\
\text { patients }(n=2) \text {, asymptomatic and } \\
\text { oncology patients }(n=11)\end{array}$ & Thyroid & PET, PET/CT & 197386 & 3659 \\
\hline Treglia 2014 & 20 & $\begin{array}{l}\text { Oncology patients }(n=16) \text {, asymptomatic } \\
\text { patients }(n=2) \text {, unclear }(n=2)\end{array}$ & Colon & PET, PET/CT & 16215 & 329 \\
\hline Kousgaard 2017 & 26 & Oncology patients $(n=26)$ & Colon & PET, PET/CT & 108578 & 2121 \\
\hline Treglia 2015 & 11 & $\begin{array}{l}\text { Known or suspected cancer }(n=7) \text {, } \\
\text { asymptomatic and oncology patients } \\
(n=4)\end{array}$ & Parotid & PET, PET/CT & 50099 & 210 \\
\hline Bertagna 2014 & 9 & Known or suspected cancer $(n=9)$ & Breast & PET, PET/CT & 96557 & 292 \\
\hline Bertagna 2014 & 6 & $\begin{array}{l}\text { Known or suspected cancer }(n=2) \text {, } \\
\text { asymptomatic and oncology patients } \\
(n=4)\end{array}$ & Prostate & PET, PET/CT & 47925 & 711 \\
\hline Xiong 2005 & 11 & $\begin{array}{l}\text { Asymptomatic }(n=2) \text {, colonic symptoms } \\
\text { for investigation }(n=9)\end{array}$ & Extra-colon & CT colonoscopy & 2797 & 998 \\
\hline Morris 2009 & 15 & Asymptomatic patients $(n=15)$ & Brain & Brain MRI & 19458 & 1864 \\
\hline Takashima 2017 & 10 (12 cohorts) & Asymptomatic $(n=11)$, psychiatric $(n=1)$ & $\begin{array}{l}\text { Brain }(n=11), \text { extra-cerebral } \\
(n=1)\end{array}$ & Brain MRI & 7858 & 2661 \\
\hline Dunet 2016 & 11 & Undergoing cardiac MRI $(\mathrm{n}=11)$ & Extra-cardiac & Cardiac MRI & 6722 & 1916 \\
\hline Flor 2013 & 16 & $\begin{array}{l}\text { Asymptomatic patients }(n=1) \text {, suspected } \\
\text { CAD }(n=13) \text {, mixed }(n=2)\end{array}$ & Extra-cardiac & Cardiac CT & 14409 & 4922 \\
\hline Dentali 2010 & 6 & $\begin{array}{l}\text { Patients not suspected to have } \\
\text { pulmonary embolism }(n=5) \text {, oncology } \\
\text { patient }(n=1)\end{array}$ & $\begin{array}{l}\text { Pulmonary vasculature } \\
\text { (pulmonary embolism) }\end{array}$ & CT chest & 6498 & 147 \\
\hline Donadini 2014 & 3 & Asymptomatic oncology patients $(n=3)$ & $\begin{array}{l}\text { Pulmonary vasculature } \\
\text { (pulmonary embolism) }\end{array}$ & CT chest & 1845 & 24 \\
\hline Jacobs 2008 & 6 & $\begin{array}{l}\text { Asymptomatic }(n=3) \text {, suspected CAD } \\
(n=3)\end{array}$ & $\begin{array}{l}\text { Thorax, abdomen, spine and } \\
\text { heart }(n=3) \text { Extra-cardiac } \\
(n=3)\end{array}$ & $\begin{array}{l}\text { CT chest }(n=3) \text {, } \\
\text { cardiac } C T(n=3)\end{array}$ & 7179 & 3272 \\
\hline Ramadorai 2014 & 7 & Asymptomatic children $(n=7)$ & Spine & MRI spine & 557 & 129 \\
\hline
\end{tabular}

PET/computed tomography (whole body PET, PET/ computed tomography: one meta-analysis excluded patients with cancer and one included patients with cancer).

Table 3 reports the meta-analysed percentage of patients with a detected incidentaloma for each imaging test. The percentage was highest in chest computed tomography ( $45 \%, 95 \%$ confidence interval $36 \%$ to $55 \%$ ), followed by computed tomography colonoscopy (38\%, 21\% to 57\%) and cardiac MRI $(34 \%, 22 \%$ to $46 \%)$. It was lowest in whole body PET or PET/computed tomography (2\%, 1\% to $4 \%$ ) and chest computed tomography for incidental pulmonary embolism $(2 \%, 1 \%$ to $4 \%)$. Intermediate rates of incidentalomas occurred in MRI of the spine $(22 \%, 19 \%$ to $26 \%)$ and MRI of the brain $(22 \%$, $14 \%$ to $31 \%)$. We did not identify any eligible systematic reviews or meta-analyses quantifying the prevalence of incidentalomas on any radiography or ultrasonography test.

\section{Malignancy in incidentalomas}

We conducted 11 meta-analyses quantifying the percentage of incidentalomas that were malignant (appendix 3). The median number of primary studies included in meta-analyses was 11 (range 2-36), the median number of incidentalomas was 771 (76-3659), and the median number of malignant incidentalomas was 68 (4-477). Our meta-analyses covered 10 different organs (table 4).
Table 4 reports the meta-analysed percentage of incidentalomas that were malignant. Results varied substantially between organs; the highest percentage of malignant incidentalomas occurred in the breast (42\%, 95\% confidence interval 31\% to 54\%), followed by the ovary $(28 \%, 11 \%$ to $48 \%)$. Prostatic $(11 \%, 1 \%$ to $28 \%)$ and colonic $(17 \%, 12 \%$ to $21 \%)$ incidentalomas were malignant between $10 \%$ and $20 \%$ of the time, whereas malignancy in incidentalomas of the brain ( $0 \%, 0 \%$ to $0.0001 \%)$, parotid $(5 \%, 2 \%$ to $10 \%)$, and adrenal gland $(0.0007 \%, 0 \%$ to $0.5 \%)$ were rare. Notably, our meta-analyses for colon, parotid, and prostate included patients with cancer because the main analysis (excluding patients with cancer) was not possible as there was only one eligible primary study (colon) or no eligible primary studies (parotid and prostate). We produced two meta-analyses estimating the percentage of thyroid incidentalomas that were malignant: a primary analysis including patients without a history of cancer and a sensitivity analysis including patients with known or suspected cancer. The primary analysis estimated a prevalence of malignancy of $28 \%$ ( $20 \%$ to $37 \%$ ), whereas the sensitivity analysis, with more power, estimated $19 \%$ (15\% to $24 \%$ ).

We did not conduct meta-analyses for malignancy on data from three systematic reviews ${ }^{83} 38$ because incidentalomas were not stratified by malignancy (either stratified by "urgency level" ${ }^{34}$ or "significant findings" ${ }^{38}$ ) or the diagnosis of incidentalomas was 


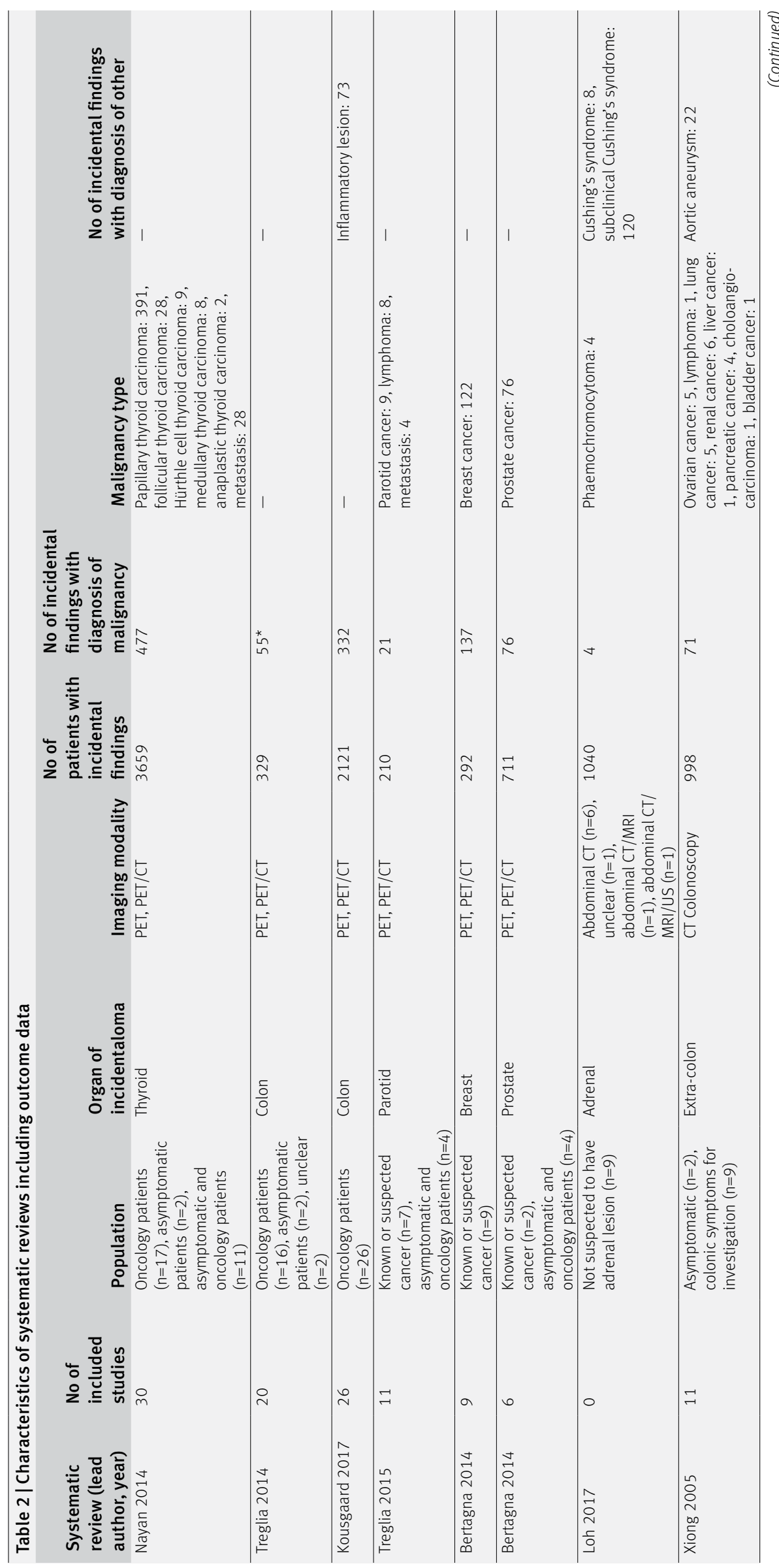

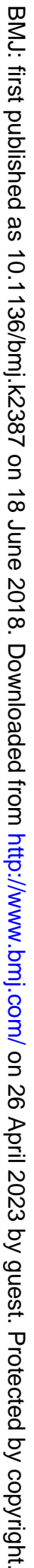




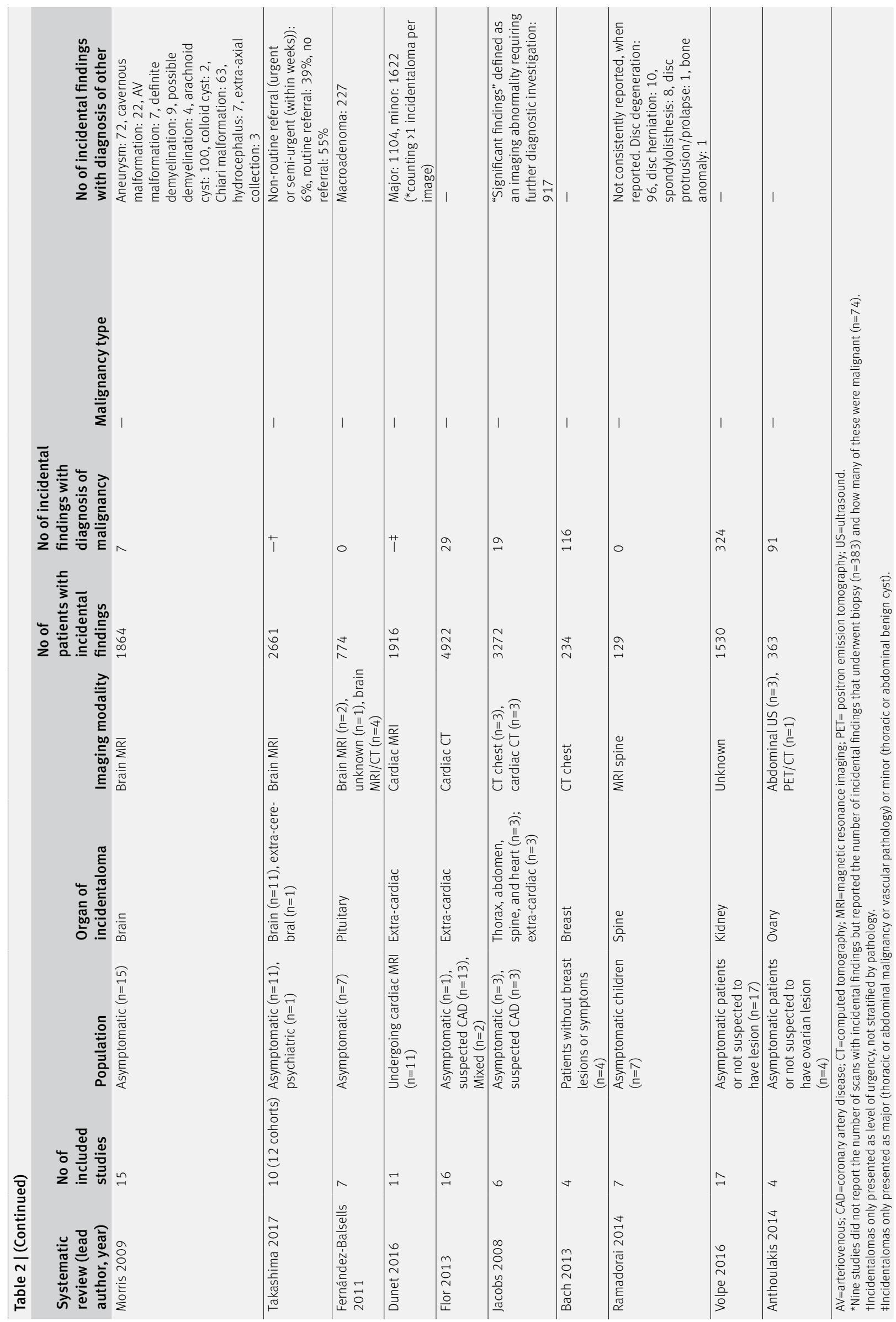

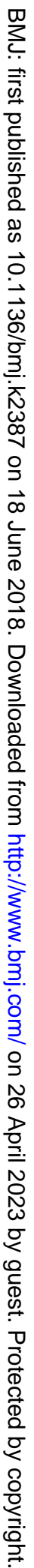




\begin{tabular}{|c|c|c|c|c|c|c|c|c|}
\hline Imaging request & $\begin{array}{l}\text { Organ of } \\
\text { incidentaloma }\end{array}$ & $\begin{array}{l}\text { No of } \\
\text { systematic } \\
\text { reviews } \neq\end{array}$ & $\begin{array}{l}\text { No of } \\
\text { primary } \\
\text { studies }\end{array}$ & $\begin{array}{l}\text { No of patients with } \\
\text { incidentalomas }\end{array}$ & $\begin{array}{l}\text { Meta-analysed } \\
\text { prevalence of } \\
\text { incidentalomas (\%; } \\
95 \% \mathrm{Cl})\end{array}$ & $\tau^{2}$ & $\begin{array}{l}1^{2} \\
(\%)\end{array}$ & $\begin{array}{l}\text { Proportion (\%) of } \\
\text { heterogeneity explained } \\
\text { by study size }\left(\mathrm{R}^{2} \text { via }\right. \\
\text { meta-regression) }\end{array}$ \\
\hline MRI spine & Spine & 1 & 7 & 129 & 22 (19 to 26$)$ & 0 & 0 & 0 \\
\hline Cardiac MRI & Extra-cardiac & 1 & 11 & 1916 & $34(22$ to 46$)$ & 0.05 & 99 & 42 \\
\hline $\begin{array}{l}\text { CT chest (including } \\
\text { CT cardiac) }\end{array}$ & $\begin{array}{l}\text { Thorax, abdomen, } \\
\text { spine, heart }\end{array}$ & 2 & 22 & 8194 & 45 (36 to 55$)$ & 0.05 & 99 & 0 \\
\hline CT chest* & $\begin{array}{l}\text { Pulmonary vasculature } \\
\text { (pulmonary embolism) }\end{array}$ & 2 & 5 & 137 & 2 (1 to 4$)$ & 0.002 & 91 & 0 \\
\hline CT chest† & $\begin{array}{l}\text { Pulmonary vasculature } \\
\text { (pulmonary embolism) }\end{array}$ & 2 & 9 & 171 & 2 (1 to 3) & 0.002 & 88 & 0 \\
\hline CT colonoscopy & Extra-colon & 1 & 11 & 998 & 38 (21 to 57) & 0.09 & 99 & 0 \\
\hline MRI brain & Brain & 2 & 27 & 4525 & 22 (14 to 31$)$ & 0.07 & 100 & 0.22 \\
\hline $\begin{array}{l}\text { Whole body PET, } \\
\text { PET/CT* }^{*}\end{array}$ & Thyroid, colon & 2 & 3 & 133 & 2 (1 to 4$)$ & 0.003 & 97 & 95 \\
\hline $\begin{array}{l}\text { Whole body PET, } \\
\text { PET/CT† }\end{array}$ & $\begin{array}{l}\text { Thyroid, colon, parotid, } \\
\text { breast, prostate }\end{array}$ & 6 & 93 & 7322 & 0.25 (2 to 3$)$ & 0.004 & 99 & 0.25 \\
\hline \multicolumn{9}{|c|}{$\begin{array}{l}\text { CT=computed tomography; } \mathrm{MRI}=\text { magnetic resonance imaging; } \mathrm{PET}=\text { positron emission tomography. } \\
\text { *Analysis only includes patients without a history of cancer. } \\
\text { †Sensitivity analysis including patients with known or suspected cancer. } \\
\text { ‡Fifteen systematic reviews provided data to quantify the prevalence of detecting incidentalomas. The two results from whole body PET-PET/computed tomography overlap, }+ \text { is the } \\
\text { sensitivity analysis). }\end{array}$} \\
\hline
\end{tabular}

inconsistently or not reported in primary studies. ${ }^{8}$ One of these systematic reviews quantified the outcome of brain incidentalomas. ${ }^{34}$ of 2082 patients with brain incidentalomas, around half $(\mathrm{n}=1146,55 \%)$ did not require a referral, whereas $815(39 \%)$ were routinely referred for follow-up. For the remaining 121 patients (6\%), urgent or semi-urgent referral was required. The second systematic review ${ }^{38}$ quantified the outcome of incidentalomas of the chest; both the proportion of "significant findings," which was defined as an abnormality requiring further diagnostic investigation, and the rate of malignancy (but did not state in which organ the malignancies occurred). They reported that $19(0.6 \%)$ incidentalomas noted on chest computed tomography scans were definitively malignant, whereas 917 (28\%) incidentalomas required further diagnostic investigation. The remaining systematic review $^{8}$ also reported that $29(0.6 \%)$ incidentalomas found on cardiac computed tomography were malignant without specifying the organs involved.

\section{Other outcomes from incidentalomas}

Six systematic reviews report the non-malignancy outcomes of incidentalomas ${ }^{73} 394344$ (table 2). The outcomes of the incidentalomas noted on MRIs of the spine of children were inconsistently reported, but all reported were non-cancerous, with the majority reported as disc degeneration $(n=96,74 \%)$ followed by disc herniation $(n=10,8 \%)$. Around $30 \%(n=227)$ of pituitary incidentalomas were macroadenomas, whereas brain incidentalomas more broadly were commonly arachnoid cysts ( $\mathrm{n}=100,5.4 \%)$, aneurysms $(n=72,3.9 \%)$, or Chiari malformations $(n=63,3.4 \%)$. Extra-colonic incidentalomas noted on computed tomography colonoscopy were diagnosed as aortic aneurysms 2.2\% $(\mathrm{n}=22)$ of the time. Around $12 \%$ $(n=120)$ of patients with incidental adrenal lesions had subclinical Cushing's syndrome, but less than $1 \%(\mathrm{n}=8)$ had Cushing's syndrome itself. Lastly, 3.4\% $(n=73)$ of colonic incidentalomas were non-cancerous inflammatory lesions.

\begin{tabular}{|c|c|c|c|c|c|c|c|}
\hline $\begin{array}{l}\text { Organ of } \\
\text { incidentaloma }\end{array}$ & $\begin{array}{l}\text { No of systematic } \\
\text { reviews }\end{array}$ & $\begin{array}{l}\text { No of primary } \\
\text { studies }\end{array}$ & No of incidentalomas & $\begin{array}{l}\text { Meta-analysed proportion of } \\
\text { malignancies }(\% ; 95 \% \mathrm{Cl})\end{array}$ & $\tau^{2}$ & $I^{2}(\%)$ & $\begin{array}{l}\text { Proportion (\%) of heterogeneity } \\
\text { explained by study size ( } \mathrm{R}^{2} \text { via meta- } \\
\text { regression) }\end{array}$ \\
\hline Adrenal & 1 & 9 & 1040 & 0.0007 (0 to 0.5$)$ & 0 & 0 & NA \\
\hline Ovary & 1 & 5 & 363 & 28 (11 to 48$)$ & 0.05 & 94 & 76 \\
\hline Brain & 1 & 15 & 1864 & 0 (0 to 0.0001$)$ & 0 & 0 & NA \\
\hline Breast & 2 & 17 & 661 & $42(31$ to 54$)$ & 0.04 & 85 & 0 \\
\hline Extra-colonic & 1 & 11 & 998 & 14 (4 to 28$)$ & 0.07 & 95 & 11 \\
\hline Renal & 1 & 16 & 1530 & 25 (16 to 34$)$ & 0.04 & 94 & 0.7 \\
\hline Thyroid* & 1 & 2 & 111 & $28(20$ to 37$)$ & 0 & 0 & NA \\
\hline Thyroidt & 1 & 31 & 3659 & 19 (15 to 24$)$ & 0.02 & 89 & 42 \\
\hline Colon $\neq$ & 2 & 36 & 661 & 17 (12 to 21$)$ & 0.02 & 86 & 7 \\
\hline Parotid§ & 1 & 11 & 210 & $5(2$ to 10$)$ & 0 & 0 & NA \\
\hline Prostate§ & 1 & 6 & 771 & 11 (1 to 28$)$ & 0.005 & 69 & 0 \\
\hline \multicolumn{8}{|c|}{$\begin{array}{l}\text { NA=not applicable. } \\
\text { *Analysis only includes asymptomatic patients without a history of cancer. } \\
\text { tSensitivity analysis including patients with known or suspected cancer; } 5.9 \% \text { of these cancers metastasised to the thyroid (28 of 477), and systematic review authors did not provide the number } \\
\text { of metastases per primary study, thus it was not possible to exclude them from meta-analysis. } \\
\text { †Main analysis was not possible because only one primary study included patients who did not have cancer. This analysis included patients with known or suspected cancer. Systematic review } \\
\text { authors did not state whether their data included or excluded metastasis, but metastasis to colon is rare. }\end{array}$} \\
\hline
\end{tabular}


Overall evidence assessment: meta-analyses of prevalence of incidentalomas

Of the nine meta-analyses estimating the prevalence of incidentalomas across imaging tests, only one had an $\mathrm{I}^{2}$ value lower than $50 \%$ (MRI of the spine). After meta-regression, one meta-analysis had an $\mathrm{R}^{2}$ value greater than $50 \%$ (whole body PET, PET/computed tomography (including patients without a history of cancer)). Neither of these two meta-analyses had $>1000$ incidentalomas. Four meta-analyses had more than 1000 incidentalomas (cardiac MRI, chest computed tomography, MRI of the brain, and whole body PET, PET/computed tomography; sensitivity analysis). Sensitivity analyses with up to one outlying study excluded did reduce the $\mathrm{I}^{2}$ values in all metaanalyses with an originally high $\mathrm{I}^{2}$, but none reached lower than $50 \%$. Thus, none of our prevalence metaanalyses met both our predefined thresholds $\left(\mathrm{I}^{2}<50 \%\right.$ and $>1000$ incidentalomas) for consistency and amount of evidence.

\section{Overall evidence assessment: meta-analyses of malignancy}

Of the 11 meta-analyses estimating the proportion of malignant lesions among detected incidentalomas across organs, four had $\mathrm{I}^{2}$ values lower than 50\% (adrenal, brain, thyroid, and parotid). After metaregression, one meta-analysis had an $\mathrm{R}^{2}$ value greater than 50\% (ovary), and one further meta-analysis had an $\mathrm{R}^{2}$ of $42 \%$ (thyroid (for patients with cancer)). Of these six meta-analyses, two had more than 1000 incidentalomas (adrenal and thyroid (for patients with cancer)). Of the five remaining meta-analyses, a sensitivity analysis excluding an outlying study reduced the $\mathrm{I}^{2}$ value to lower than $50 \%$ in one metaanalysis (prostate), although this meta-analysis contained only 771 incidentalomas. Thus, one metaanalysis (adrenal) reached both our predefined thresholds ( $\mathrm{I}^{2}<50 \%$ and $>1000$ incidentalomas) for consistency and amount of evidence.

\section{Quality assessment: AMSTAR}

Appendix 4 shows the AMSTAR assessment for the 20 included systematic reviews. The median AMSTAR score was 5/11 (range 2-10). Notably, systematic reviews consistently failed to report excluded studies (1/20 systematic reviews achieved this) and a prespecified protocol $(3 / 20)$.

\section{Discussion}

We present an umbrella review quantifying the prevalence of incidentalomas stratified by imaging scan and outcomes of incidentalomas stratified by organ. Our results summarise data for six imaging tests and 12 organs.

\section{Principal findings}

Computed tomography of the chest, computed tomography colonoscopy, and cardiac MRI scans had the highest prevalence of incidentalomas. Breast incidentalomas had the highest rate of malignancy, whereas brain and adrenal incidentalomas had notably low rates of malignancy. Very large heterogeneity (in terms of the $\mathrm{I}^{2}$ metric) was very common in these data. Few meta-analyses had consistent, non-heterogeneous evidence and a large amount of data.

\section{Implications}

Clinicians

Our results provide essential information for two common and important clinical tasks: ordering an imaging test and further management for a patient with an incidental finding. Before clinicians order an imaging test, they should weigh up the benefits and risks. Imaging tests have many risks; there is the risk of radiation exposure from computed tomography and radiography ${ }^{46}$ and the risk of allergic reactions and nephropathy from the contrast dye used in some computed tomography and MRI scans. ${ }^{47} 48$ With the increasing use and sensitivity of imaging technology, there is also the risk of incidental findings. Incidentalomas can cause patient anxiety ${ }^{10}$ and can lead to further investigation and treatment, some of which may cause more harm than good. Incidentalomas also have financial consequences; there are costs associated with further patient management, but also the potential change to a patient's insurance status and premiums. ${ }^{49}$ Concern surrounding these potential consequences has led to guidance encouraging clinicians to discuss the risk of incidental findings with their patients before they order an imaging test. ${ }^{4}$ Our results-the quantification of the prevalence of incidental findings from different imaging testsequips clinicians to appropriately inform patients about the risks of incidentalomas before the ordering of a scan. Our study provides the data for clinicians to quantify the risk of incidentalomas for numerous different imaging tests and thus allow clinicians and patients to weigh up the risks and benefits of undergoing an imaging test.

Our results also equip clinicians to make evidence based decisions regarding the management of patients with incidentalomas. Clinicians who routinely request images have expressed uncertainty regarding further investigations and/or treatment of incidentalomas. ${ }^{12}$ Clinicians, particularly primary care physicians, are unsure which incidentalomas require urgent further investigation, nor are they sure which incidentalomas are likely to be benign. ${ }^{12}$ As such, there have been calls for "more research to investigate potential outcomes (of incidentalomas)." ${ }^{4}$ Our study helps meet these calls. For 12 different organs, we provide the prevalence of malignancy from incidentalomas, and list the other, non-malignancy outcomes. These data will reduce uncertainty surrounding clinical management of incidentalomas and thus help clinicians make evidence based management decisions. Our results will also help clinicians adequately inform patients about the likely outcomes from incidental findings. For instance, our results provide clinicians with the data to confidently reassure patients that an adrenal incidentaloma is likely to be benign. Our results 
similarly equip clinicians to urgently investigate a patient with an incidental finding of the breast. Although our results can equip clinicians and patients to make evidence baseddecisions regarding the management of incidental findings, clinicians should interpret the available evidence in the context of the individual patient.

Lastly, our results can be used to support evidence based guidelines concerning the management of incidental findings. New and updated guidelines can reduce uncertainty in the management of incidental findings internationally. We elaborate on the guideline implications from our results in the Policy makers section below.

\section{Patients}

Healthcare is moving towards a shared decision making model. ${ }^{5051}$ In this patient centred approach, diagnostic and treatment decisions are made collaboratively between clinician and patient. A major barrier to achieving shared decision making is the difficulty informing patients appropriately of the intricacies and complexity of clinical decisions. ${ }^{52}$ One solution to duly inform patients is evidence based decision guides. ${ }^{53}$ Ideally, these guides should inform patients of the chance of an incidental finding from an imaging scan, and might also explain the likely outcomes of different incidentalomas. Our study provides the data to fill these decision guidelines and thus adequately inform patients. This could facilitate a truly shared decision on whether to undergo an imaging scan and help decide the next steps for handling an incidentaloma.

\section{Policy makers}

Our results are relevant for policy makers in two ways: to inform new guidelines about the management of organ specific incidentalomas, and to update existing guidelines. We report the outcomes of incidentalomas across 12 different organs (table 2), as well as the meta-analysed prevalence of malignancy (table 4). Guidelines exist in the United States and Europe for the management of incidentalomas for five organs (pituitary, ${ }^{1}$ adrenal, ${ }^{13}{ }^{14}$ ovary, ${ }^{15}$ kidney, ${ }^{17}{ }^{54}$ and thyroid $\left.^{16}\right)$. Guidelines concerning pituitary and adrenal incidentalomas offer recommendations for clinicians requesting imaging, whereas the remaining three guidelines (ovary, ${ }^{15}$ renal, $^{17}{ }^{54}$ and thyroid ${ }^{16}$ ) target radiologists and their recommendations assume a level of radiological expertise.

The American Association of Clinical Endocrinologists and American Association of Endocrine Surgeons 2009 guideline on the management of adrenal incidentalomas ${ }^{14}$ targets nonradiologist clinicians and recommends: "Patients with an adrenal incidentaloma should undergo evaluation clinically, biochemically, and radiographically for signs and symptoms of hypercortisolism, aldosteronism (if hypertensive), the presence of a pheochromocytoma, or a malignant tumour." ${ }^{13}$ This recommendation appears congruent with our results; our data suggest that around $12 \%$ of patients with adrenal incidentalomas will have subclinical Cushing's syndrome (also known as low level autonomous cortisol secretion). However, the same guideline recommends: "Patients with adrenal incidentalomas who do not fulfil the criteria for surgical resection need to have radiographic re-evaluation at 3 to 6 months and then annually for 1 to 2 years."13 This recommendation is incongruent with our data: we found that less than $1 \%$ of adrenal incidentalomas are malignant. The more recent 2016 European Society of Endocrinology recommendations ${ }^{14}$ are more closely aligned with our results, but are also somewhat conservative: "We recommend that all adrenal incidentalomas undergo an imaging procedure to determine if the mass is homogeneous and lipid rich and therefore benign. For this purpose, we primarily recommend the use of non-contrast computed tomography. We suggest that if the non-contrast computed tomography is consistent with a benign adrenal mass (Hounsfield units $\leq 10$ ) that is homogeneous and smaller than $4 \mathrm{~cm}$, no further imaging is required." 14

Our results suggest that about $30 \%$ of patients with pituitary incidentalomas are diagnosed with macroadenomas. The joint guideline from the Endocrine Society (USA) and European Society of Endocrinology on the management of pituitary incidentaloma ${ }^{1}$ aligns with our data. It states: "We recommend that patients with incidentalomas not meeting criteria for surgical removal be followed with clinical assessments, neuroimaging (MRI at six months for macroincidentalomas, one year for a microincidentaloma, and thereafter progressively less frequently if unchanged in size), visual field examinations for incidentalomas that abut or compress the optic nerve and chiasm (six months and yearly), and endocrine testing for macroincidentalomas (six months and yearly) after the initial evaluations." 1

It is difficult to compare our results with the three remaining organ guidelines that offer management recommendations based on features that require radiologist interpretation. ${ }^{16} 17 \quad 5455$ Although we acknowledge the necessity of radiologist specific guidelines, our results for these organs (ovary, thyroid, and kidney) could be used to fill an essential gap: guidelines targeted at the requesting physician.

Similarly, to our knowledge, no US or European guidelines exist for the management of incidental findings of the spine, breast, brain, colon, prostate, or parotid. Guidelines for these lesions are essential, since requesting physicians often feel uncertain about how to best manage incidental findings. ${ }^{12}$ Furthermore, the number of imaging scans continues to increase, ${ }^{56}$ as does the resolution of scan images and the number of slices per scan. ${ }^{11}$ The umbrella review can offer the underpinning evidence of these guidelines.

\section{Strengths and weaknesses in relation to other studies}

The relative merits of the umbrella review approach have been described in detail. ${ }^{18} 192957$ A strength of our study is the stratification of our results by imaging 
scan and organ that could enhance value to clinicians. We used a broad, systematic search and a standardised meta-analysis method, and considered evidence on all types of imaging tests and organs. Published systematic reviews used inconsistent and often inappropriate methods to synthesise data. For instance, none of the included systematic reviews used the inverse variance method in their meta-analyses, nor did any use double arcsine transformation to stabilise the variance, as recommended. ${ }^{21}$ We extracted data from included systematic reviews and primary studies, but performed our own, independent meta-analyses using these more appropriate methods

A limitation of our analyses is the frequently very high levels of suspected heterogeneity $\left(\mathrm{I}^{2}\right)$. Fifteen of our 20 meta-analyses had $\mathrm{I}^{2}$ values greater than $50 \%$, which meta-regression of sample size only partly explained in three of these 15 cases. While these values of heterogeneity seem extreme, one should note that in most cases where very large $\mathrm{I}^{2}$ values were detected, this was seen because several single studies had seemingly very high precision, thus even with modest absolute differences in prevalence or proportion among themselves, they would yield very large $\mathrm{I}^{2}$ values. Although we had not anticipated this in our original preregistered protocol, in these cases it may be best to examine the absolute magnitude of the differences between studies. This is reflected best by the between-study variance $\left(\tau^{2}\right)$ values, and 18 of the 20 meta-analyses had values of 0.05 or less (table 3, table 4, and appendices). Such values are not uncommon in meta-analyses. ${ }^{58}$

A major contributor to the observed levels of heterogeneity is different definitions used by radiologists to classify and report the presence of an incidentaloma. Similar to the concept of varying thresholds for defining a test result positive in metaanalyses of diagnostic accuracy, heterogeneity is common when primary study authors use different definitions for "positive," 59 or more specifically in our case, varying thresholds for what constitutes an incidentaloma. Unfortunately, there are no universally accepted definitions or thresholds for what constitutes an incidentaloma. ${ }^{60}$ Thus, our results mirror the likely situation clinicians face when requesting imaging scans: the same radiologist is unlikely to report all images requested and thus there is likely to be heterogeneity in the reporting of incidentalomas from image to image.

Additional factors likely to have contributed to the observed heterogeneity include variation in baseline patient characteristics, imaging protocols, thickness and number of imaging slices, and histological follow-up to confirm or exclude malignancy between primary studies included in meta-analyses. Varying imaging protocols between primary studies was a likely contributor to the high heterogeneity in the chest computed tomographymeta-analyses (both theanalysis focused on prevalence of pulmonary embolism, and the other focused on any other incidentaloma). For the pulmonary embolism meta-analysis, primary studies used varying slice thickness; from $1.25 \mathrm{~mm}$ to $10 \mathrm{~mm}$ (the number of slices was also varied between primary studies but was less consistently reported). This was similar for the non-pulmonary embolism metaanalysis, where the imaging procedure varied within one of the included systematic reviews. ${ }^{38}$ Four primary studies used electron beam computed tomography, five multi-slice computed tomography, and two single slice computed tomography (the other included systematic review $^{8}$ did not provide data to address this). Similar discrepancies in imaging protocols exist for the brain MRI meta-analysis: around half the included studies used high resolution scans, the remaining used low resolution (or not reported). In the computed tomography colonoscopy meta-analysis, three of the included primary studies used contrast (27\%), the remaining did not. Lastly, the variable duration and type of follow-up and perusal of incidentalomas by primary study authors also likely contributed to heterogeneity through diagnostic investigative bias.

A limitation of the meta-analyses concerning the prevalence of incidentalomas is the possibility of counting more than one incidentaloma per image. Of the 15 systematic reviews contributing to the nine meta-analyses of prevalence, most $(n=12,80 \%)$ reported only one incidentaloma counted per patient. Of the remaining three, this was unclear for two systematic reviews, ${ }^{79}$ but interrogation of primary studies within these systematic reviews revealed that most of the primary studies within these systematic reviews counted only one incidentaloma per patient $\left(71 \%{ }^{39}\right.$ and $100 \%{ }^{7}$ respectively). The remaining systematic review ${ }^{28}$ did appear to count more than one incidentaloma per patient. However, this limitation does not affect the results or our conclusions. The systematic review that counted more than one incidentaloma per patient contributed data to the meta-analysis quantifying incidentaloma prevalence in whole body PET scans. Excluding this systematic review's data from the meta-analysis in question did not substantially change the meta-analysed estimate (2.46\%, 95\% confidence interval, $1.96 \%$ to $3.01 \%$ ) versus $2.53 \%$ (95\% confidence interval $2.13 \%$ to $2.97 \%)$ nor the $\mathrm{I}^{2}$ value $(98.8 \% v 98.9 \%)$.

Lumbreras and colleagues ${ }^{61}$ conducted a systematic review and meta-analysis quantifying the "frequency of incidental findings" from imaging tests. They combined data from all imaging modalities (radiography, computed tomography, MRI, etc) and presented their results collectively-they estimated that around $25 \%$ of imaging tests return an incidentaloma. We chose to present our results stratified by the specific imaging test (eg, computed tomography scan of the chest) rather than for all imaging tests and modalities together, because we considered it more useful for clinicians and patients when ordering a specific test. Lumbreras and colleagues did not quantify the outcomes of incidentalomas. A further strength of our study includes a sensitivity and detailed search strategy: we screened over 7000 titles and abstracts and included 240 primary studies, compared with 
the Lumbreras study, which screened 250 and included 44 primary studies. We also performed, as recommended for percentage data, double arcsine transformation to stabilise the variance, ${ }^{21}$ using the inverse variance method, ${ }^{22}$ and used a random effects model for our meta-analyses. The review by Lumbreras and colleagues calculated the mean "frequency of incidental findings" without meta-analysis models or double arcsine transformation. Furthermore, we should caution that our umbrella review does not capture data on some of the downstream effects of having an incidentaloma, for example, the number of follow-up scans and treatments used, how necessary these management steps are, and whether patients gain or lose eventually in terms of survival or other major outcomes. Hard clinical endpoints would require randomised trials to assess reliably, and utilisation of services may be also context dependent and vary across healthcare systems.

Lastly, as is consistent with umbrella review methodology, 18192957 primary studies not captured within our included systematic reviews are not included and we did not assess the quality of primary studies, but trusted the choice of the original systematic reviewers to consider them eligible for inclusion.

\section{Future research and next steps}

Our review highlights some clear gaps in the literature. We found no systematic reviews quantifying the prevalenceofincidentalomasonanytypeof radiography (x ray) or ultrasonography. Similarly, future systematic reviews addressing the outcomes of incidentalomas of the liver, pancreas, lungs, and spleen would be advantageous. Further, given the emerging evidence that orthopaedic procedures of degenerative joints are of limited benefit, ${ }^{62}{ }^{63}$ systematic reviews quantifying the prevalence and outcome of incidentalomas from imaging of the joint (eg, knees, hips, shoulders) would be valuable. Furthermore, guidelines stipulating what constitutes an incidentaloma and what does not, stratified by organ, would be advantageous for clinicians, radiologists, and researchers. Efforts from radiological professional societies to standardise definitions of incidentaloma thresholds, especially for organs where we documented very large heterogeneity, would be useful. Lastly, incidentalomas of the breast and thyroid had the highest rates of malignancy. Cancers of both these organs are known to be commonly overdiagnosed $^{64-66}$ and it is plausible that incidentally diagnosed breast and thyroid cancers might represent (in part) overdiagnosis. Long term follow-up of breast and thyroid cancers diagnosed incidentally would be advantageous to quantify how likely incidentally diagnosed cancers are non-progressive or never grow to cause a patient harm.

\section{Conclusions}

Data from our comprehensive umbrella review conclude that computed tomography of the chest, computed tomography colonoscopy, and cardiac MRI imaging scans have the highest frequencies of detecting incidentalomas. Of the 10 organs with supporting data, breast, ovarian, and thyroid incidentalomas have the highest rates of malignancy. This umbrella review will help clinicians and patients weigh up the pros and cons of requesting imaging scans, and will assist with management decisions after an incidentaloma diagnosis. Our results can underpin the creation of guidelines to assist clinicians and patients through these decisions.

Acknowledgements: We thank Ben Feakins for his help throughout the study.

Contributors: JWOS and II conceived and designed the study, and interpreted the results. TM, SG, and JWOS screened titles, abstracts, and full texts. JWOS and SG performed the data extraction. JWOS did the statistical analysis and JWOS is the study guarantor. All authors reviewed the manuscript. The corresponding author attests that all listed authors meet authorship criteria and that no others meeting the criteria have been omitted.

Funding: This study did not receive any specific funding. The MetaResearch Innovation Center at Stanford (METRICS) has been funded by the Laura and John Arnold Foundation.

Competing interests: All authors have completed the ICMJE uniform disclosure form at www.icmje.org/coi_disclosure.pdf and declare: no support from any organisation for the submitted work; no financial relationships with any organisations that might have an interest in the submitted work in the previous three years; no other relationships or activities that could appear to have influenced the submitted work.

Ethical approval: Not required.

Data sharing: We extracted data from published systematic reviews, all of which are available and accessible.

The manuscript's guarantor affirms that the manuscript is an honest, accurate, and transparent account of the study being reported; that no important aspects of the study have been omitted; and that any discrepancies from the study as planned (and, if relevant, registered) have been explained.

This is an Open Access article distributed in accordance with the terms of the Creative Commons Attribution (CC BY 4.0) license, which permits others to distribute, remix, adapt and build upon this work, for commercial use, provided the original work is properly cited. See: http://creativecommons.org/licenses/by/4.0/

1 Freda PU, Beckers AM, Katznelson L, et al, Endocrine Society. Pituitary incidentaloma: an endocrine society clinical practice guideline. J Clin Endocrinol Metab 2011;96:894-904. doi:10.1210/ jc.2010-1048.

2 Young WF Jr. Clinical practice. The incidentally discovered adrenal mass. N Engl J Med 2007;356:601-10. doi:10.1056/ NEJMcp065470

3 Hitzeman N, Cotton E. Incidentalomas: initial management. Am Fam Physician 2014;90:784-9.

4 Weiner C. Anticipate and communicate: Ethical management of incidental and secondary findings in the clinical, research, and direct-to-consumer contexts (December 2013 report of the Presidential Commission for the Study of Bioethical Issues). Am J Epidemiol 2014;180:562-4. doi:10.1093/aje/kwu217.

5 Smith-Bindman R, Miglioretti DL, Larson EB. Rising use of diagnostic medical imaging in a large integrated health system. Health Aff (Millwood) 2008;27:1491-502. doi:10.1377/ hlthaff.27.6.1491

6 O'Sullivan JW, Albasri A, Nicholson BD, et al. Overtesting and undertesting in primary care: a systematic review and meta-analysis BMJ Open 2018;8:e018557. doi:10.1136/bmjopen-2017-018557.

7 Morris Z, Whiteley WN, Longstreth WT Jr, et al. Incidental findings on brain magnetic resonance imaging: systematic review and metaanalysis. BMJ 2009;339:b3016. doi:10.1136/bmj.b3016

8 Flor N, Di Leo G, Squarza SAC, et al. Malignant incidental extracardiac findings on cardiac CT: systematic review and metaanalysis. AIR Am / Roentgenol 2013;201:555-64. doi:10.2214/ AJR.12.10306

9 Xiong T, Richardson M, Woodroffe R, Halligan S, Morton D, Lilford RJ. Incidental lesions found on CT colonography: their nature and frequency. Br J Radiol 2005;78:22-9. doi:10.1259/bjr/67998962.

10 Powell DK. Patient explanation guidelines for incidentalomas: helping patients not to fear the delayed surveillance. AJR Am / Roentgenol 2014;202:W602. doi:10.2214/AJR.13.12337.

11 Kole J, Fiester A. Incidental findings and the need for a revised informed consent process. AIR Am J Roentgenol 2013;201:1064-8. doi:10.2214/AJR.13.11138. 
12 Sexton SM. How should we manage incidentalomas? Am Fam Physician 2014;90:758-9. https://www.aafp.org/afp/2014/1201/ p758.html\#afp20141201p758-b2.

13 Zeiger MA, Thompson GB, Duh Q-Y, et al, American Association of Clinical Endocrinologists, American Association of Endocrine Surgeons. American Association of Clinical Endocrinologists and American Association of Endocrine Surgeons Medical Guidelines for the Management of Adrenal Incidentalomas: executive summary of recommendations. Endocr Pract 2009:15:450-3. doi:10.4158/ EP.15.5.450.

14 Fassnacht M, Arlt W, Bancos I, et al. Management of adrenal incidentalomas: European Society of Endocrinology Clinical Practice Guideline in collaboration with the European Network for the Study of Adrenal Tumors. Eur J Endocrinol 2016;175:G1-34. doi:10.1530/ EJE-16-0467.

15 Patel MD, Ascher SM, Paspulati RM, et al. Managing incidental findings on abdominal and pelvic CT and MRI, part 1: white paper of the ACR Incidental Findings Committee II on adnexal findings. J Am Coll Radiol 2013:10:675-81. doi:10.1016/j.jacr.2013.05.023.

16 Hoang JK, Langer JE, Middleton WD, et al. Managing incidental thyroid nodules detected on imaging: white paper of the ACR Incidental Thyroid Findings Committee. J Am Coll Radiol 2015:12:143-50, doi:10.1016/j.jacr.2014.09.038.

17 Smith AD, Remer EM, Cox KL, et al. Bosniak category IIF and III cystic renal lesions: outcomes and associations. Radiology 2012;262:15260 doi:10.1148/radiol 11110888

18 Li X, Meng X, Timofeeva M, et al. Serum uric acid levels and multiple health outcomes: umbrella review of evidence from observational studies, randomised controlled trials, and Mendelian randomisation studies. BMJ 2017;357:j2376. doi:10.1136/bmj.j2376.

19 Theodoratou E, Tzoulaki I, Zgaga L, loannidis JP. Vitamin D and multiple health outcomes: umbrella review of systematic reviews and meta-analyses of observational studies and randomised trials. BMJ 2014;348:g2035. doi:10.1136/bmj.g2035

20 Lau J, loannidis JP, Schmid CH. Quantitative synthesis in systematic reviews. Ann Intern Med 1997;127:820-6. http://annals.org/ article.aspx?articleid=710939. doi:10.7326/0003-4819-127-9199711010-00008

21 Barendregt JI, Doi SA, Lee YY, Norman RE, Vos T. Meta-analysis of prevalence. J Epidemiol Community Health 2013;67:974-8. doi:10.1136/jech-2013-203104

22 Doi SAR, Barendregt JJ, Khan S, Thalib L, Williams GM. Advances in the meta-analysis of heterogeneous clinical trials I: The inverse variance heterogeneity model. Contemp Clin Trials 2015;45(Pt A):130-8. doi:10.1016/j.cct.2015.05.009.

23 Higgins JPT, Thompson SG, Deeks JJ, Altman DG. Measuring inconsistency in meta-analyses. BMJ 2003;327:557-60. doi:10.1136/bmj.327.7414.557.

24 Higgins JPT, Thompson SG, Spiegelhalter DJ. A re-evaluation of random-effects meta-analysis. I R Stat Soc Ser A Stat Soc 2009;172:137-59. doi:10.1111/j.1467-985X.2008.00552.x.

25 IntHout J, loannidis JP, Rovers MM, Goeman JJ. Plea for routinely presenting prediction intervals in meta-analysis. $B M$ Open 2016;6:e010247. doi:10.1136/bmjopen-2015-010247.

26 Patsopoulos NA, Evangelou E, loannidis JPA. Sensitivity of between-study heterogeneity in meta-analysis: proposed metrics and empirical evaluation. Int J Epidemiol 2008;37:1148-57. doi:10.1093/ije/dyn065.

27 Shea BJ, Grimshaw IM, Wells GA, et al. Development of AMSTAR: a measurement tool to assess the methodological quality of systematic reviews. BMC Med Res Methodol 2007;7:10. doi:10.1186/1471 2288-7-10

28 Nayan S, Ramakrishna J, Gupta MK. The proportion of malignancy in incidental thyroid lesions on 18-FDG PET Study. Otolaryngol Head Neck Surg 2014;151:190-200. doi:10.1177/0194599814530861.

29 Bellou V, Belbasis L, Tzoulaki I, Evangelou E, loannidis JP. Environmental risk factors and Parkinson's disease: An umbrella review of meta-analyses. Parkinsonism Relat Disord 2016;23:1-9. doi:10.1016/j.parkreldis.2015.12.008.

30 Treglia G, Taralli S, Salsano M, Muoio B, Sadeghi R, Giovanella L. Prevalence and malignancy risk of focal colorectal incidental uptake detected by (18)F-FDG-PET or PET/CT: a meta-analysis. Radiol Oncol 2014:48:99-104 doi:10.2478/raon-2013-0035

31 Kousgaard SJ, Thorlacius-Ussing O. Incidental colorectal FDG uptake on PET/CT scan and lesions observed during subsequent colonoscopy: a systematic review. Tech Coloproctol 2017;21:521-9. doi:10.1007/s10151-017-1652-6.

32 Treglia G, Bertagna F, Sadeghi R, Muoio B, Giovanella L. Prevalence and risk of malignancy of focal incidental uptake detected by fluorine18-fluorodeoxyglucose positron emission tomography in the parotid gland: a meta-analysis. Eur Arch Otorhinolaryngol 2015;272:361726. doi:10.1007/s00405-014-3308-8.

33 Bertagna F, Treglia G, Orlando E, et al. Prevalence and clinical significance of incidental F18-FDG breast uptake: a systematic review and meta-analysis. Jpn J Radiol 2014;32:59-68. doi:10.1007/ s11604-013-0270-0
34 Takashima K, Takimoto Y, Nakazawa E, et al. Discovery and informing research participants of incidental findings detected in brain magnetic resonance imaging studies: Review and multiinstitutional study. Brain Behav 2017;7:e00676. doi:10.1002/ brb3.676.

35 Dunet V, Schwitter J, Meuli R, Beigelman-Aubry C. Incidental extracardiac findings on cardiac MR: Systematic review and metaanalysis. J Magn Reson Imaging 2016;43:929-39. doi:10.1002/ jmri.25053.

36 Dentali F, Ageno W, Becattini C, et al. Prevalence and clinical history of incidental, asymptomatic pulmonary embolism: a meta-analysis. Thromb Res 2010;125:518-22. doi:10.1016/j. thromres.2010.03.016.

37 Donadini MP, Dentali F, Squizzato A, Guasti L, Ageno W. Unsuspected pulmonary embolism in cancer patients: a narrative review with pooled data. Intern Emerg Med 2014;9:375-84 doi:10.1007/s11739-014-1066-7.

38 Jacobs PC, Mali WPTM, Grobbee DE, van der Graaf Y. Prevalence of incidental findings in computed tomographic screening of the chest: a systematic review. J Comput Assist Tomogr 2008;32:214-21. doi:10.1097/RCT.0b013e3181585ff2.

39 Ramadorai U, Hire J, DeVine JG, Brodt ED, Dettori JR. Incidental findings on magnetic resonance imaging of the spine in the asymptomatic pediatric population: a systematic review. Evid Based Spine Care / 2014;5:95-100. doi:10.1055/s-0034-1386753.

40 Anthoulakis C, Nikoloudis N. Pelvic MRI as the "gold standard" in the subsequent evaluation of ultrasound-indeterminate adnexal lesions: a systematic review. Gynecol Oncol 2014;132:661-8. doi:10.1016/j. ygyno.2013.10.022.

41 Volpe A. The role of active surveillance of small renal masses. Int J Surg 2016;36:518-24. doi:10.1016/j.ijsu.2016.06.007.

42 Bach AG, Abbas J, Jasaabuu C, Schramm D, Wienke A, Surov A. Comparison between incidental malignant and benign breast lesions detected by computed tomography: a systematic review. J Med Imaging Radiat Oncol 2013;57:529-33. doi:10.1111/1754 9485.12046.

43 Fernández-Balsells MM, Murad MH, Barwise A, et al. Natural history of nonfunctioning pituitary adenomas and incidentalomas: a systematic review and metaanalysis. J Clin Endocrinol Metab 2011:96:905-12. doi:10.1210/ic.2010-1054

44 Loh HH, Yee A, Loh HS, Sukor N, Kamaruddin NA. The natural progression and outcomes of adrenal incidentaloma: a systematic review and meta-analysis. Minerva Endocrinol 2017;:42:77-87.

45 Bertagna F, Sadeghi R, Giovanella L, et al. Incidental uptake of $18 \mathrm{~F}$-fluoro-deoxyglucose in the prostate gland. Nucl Med (Stuttg) 2014:53:249-58. doi:10.3413/Nukmed-0668-14-05.

46 Smith-Bindman R, Lipson J, Marcus R, et al. Radiation dose associated with common computed tomography examinations and the associated lifetime attributable risk of cancer. Arch Intern Med 2009:169:2078-86. doi:10.1001/archinternmed.2009.427.

47 Katzberg RW, Lamba R. Contrast-induced nephropathy after intravenous administration: fact or fiction? Radiol Clin North Am 2009; 47:789-800, v. doi:10.1016/j.rcl.2009.06.002.

48 Neptune SM, Hopper KD, Matthews YL. Risks associated with the use of IV contrast material: analysis of patients' awareness. AJR Am J Roentgenol 1994;162:451-4. doi:10.2214/ajr.162.2.8310946.

49 Booth TC, Jackson A, Wardlaw JM, Taylor SA, Waldman AD. Incidental findings found in "healthy" volunteers during imaging performed for research: current legal and ethical implications. $\mathrm{Br}$ j Radiol 2010;83:456-65 doi:101259/bir/15877332.

50 Montori VM. Big Science for patient centred care. BMJ 2017;359:j5600. doi:10.1136/bmj.j5600.

51 Berger ZD, Brito JP, Ospina NS, et al. Patient centred diagnosis: sharing diagnostic decisions with patients in clinical practice. BMJ 2017;359:j4218. doi:10.1136/bmj.j4218.

52 Elwyn G, Durand MA, Song J, et al. A three-talk model for shared decision making: multistage consultation process. BMJ 2017;359:j4891. doi:10.1136/bmj.j4891.

53 Elwyn G, Wieringa S, Greenhalgh T. Clinical encounters in the post-guidelines era. BMJ 2016;353:i3200. doi:10.1136/bmj.i3200.

54 Bosniak MA. Diagnosis and management of patients with complicated cystic lesions of the kidney. A/R Am J Roentgenol 1997:169:819-21. doi:10.2214/ajr.169.3.9275903

55 Levine D, Brown DL, Andreotti RF, et al. Management of asymptomatic ovarian and other adnexal cysts imaged at US: Society of Radiologists in Ultrasound Consensus Conference Statement. Radiology 2010;256:943-54. doi:10.1148/ radiol. 10100213

56 Busby J, Schroeder K, Woltersdorf W, et al. Temporal growth and geographic variation in the use of laboratory tests by NHS general practices: using routine data to identify research priorities. Br J Gen Pract 2013;63:e256-66. doi:10.3399/bjgp13X665224.

57 Belbasis L, Savvidou MD, Kanu C, Evangelou E, Tzoulaki I. Birth weight in relation to health and disease in later life: an umbrella review of systematic reviews and meta-analyses. BMC Med 2016:14:147 doi:10.1186/s12916-016-0692-5. 
58 Pereira TV, Patsopoulos NA, Salanti G, loannidis JP. Critical interpretation of Cochran's $Q$ test depends on power and prior assumptions about heterogeneity. Res Synth Methods 2010;1:149 61. doi:10.1002/jrsm.13.

59 Leeflang MMG. Systematic reviews and meta-analyses of diagnostic test accuracy. Clin Microbiol Infect 2014;20:105-13. doi:10.1111/1469-0691.12474

60 Paterson F, Theodoraki A, Amajuoyi A, Bouloux PM, Maclachlan J, Khoo B. Radiology reporting of adrenal incidentalomas - who requires further testing? Clin Med (Lond) 2014;14:16-21. doi:10.7861/clinmedicine.14-1-16.

61 Lumbreras B, Donat L, Hernández-Aguado I. Incidental findings in imaging diagnostic tests: a systematic review. $\mathrm{Br}$ J Radiol 2010;83:276-89. doi:10.1259/bjr/98067945.

62 Sihvonen R, Paavola M, Malmivaara A, et al, Finnish Degenerative Meniscal Lesion Study (FIDELITY) Group. Arthroscopic partial meniscectomy versus sham surgery for a degenerative meniscal tear. N Engl J Med 2013;369:2515-24. doi:10.1056/ NEJMoa1305189.
63 Wartolowska K, Judge A, Hopewell S, et al. Use of placebo controls in the evaluation of surgery: systematic review. BMJ 2014;348:g3253. doi:10.1136/bmj.g3253.

64 Jørgensen KJ, Gøtzsche PC. Overdiagnosis in publicly organised mammography screening programmes: systematic review of incidence trends. BMJ 2009;339:b2587. doi:10.1136/bmj.b2587.

65 Park S, Oh C-M, Cho H, et al. Association between screening and the thyroid cancer "epidemic" in South Korea: evidence from a nationwide study. BMJ 2016:355:i5745. doi:10.1136/bmj.i5745.

66 Brodersen J, Schwartz LM, Heneghan C, O'Sullivan JW, Aronson JK, Woloshin S. Overdiagnosis: what it is and what it isn't. BMJ Evid Based Med 2018;23:1-3. doi:10.1136/ebmed-2017-110886.

Supplementary information: Appendix 1

Supplementary information: Appendix 2

Supplementary information: Appendix 3 Supplementary information: Appendix 4 